\title{
Imaging Local Polarization and Domain Boundaries in Multiferroic $\left(\mathrm{LuFeO}_{3}\right)_{\mathrm{m}} /\left(\mathrm{LuFe}_{2} \mathrm{O}_{4}\right)_{\mathrm{n}}$ Superlattices
}

\author{
Megan E. Holtz ${ }^{1}$, Julia A. Mundy ${ }^{1}$, Jarrett A. Moyer ${ }^{2}$, Charles M. Brooks ${ }^{3}$, Hena Das ${ }^{1}$, Alejandro F. \\ Rebola $^{1}$, Robert Hovden ${ }^{1}$, Craig J. Fennie ${ }^{1}$, Peter Schiffer ${ }^{2}$, Darrell G. Schlom ${ }^{3,4}$, David A. Muller ${ }^{1,4}$ \\ 1. School of Applied and Engineering Physics, Cornell University, Ithaca, NY, USA \\ 2. Department of Physics and Materials Research Institute, University of Illinois at Urbana-Champaign, \\ Urbana, IL, USA \\ 3. Department of Materials Science and Engineering, Cornell University, Ithaca, NY, USA \\ 4. Kavli Institute at Cornell for Nanoscale Science, Ithaca, NY, USA
}

Materials that couple strong ferroelectric and ferromagnetic order hold tremendous promise for nextgeneration memory devices. However, many so-called multiferroics have properties that are either weak, emerge well below room temperature, and/or lack coupling between the electric and magnetic domains, stymieing technological applications. The atomic-scale design of new multiferroics, usually realized as heterostructures or interface phases, requires a local probe of physical properties and structure inside the material. This atomic-scale feedback on ferroelectric polarization and domain structure has helped lead us to a new strong ferrimagnet-ferroelectric with the highest known simultaneous transition temperatures. These $\left(\mathrm{LuFeO}_{3}\right)_{\mathrm{m}}\left(\mathrm{LuFe}_{2} \mathrm{O}_{4}\right)_{\mathrm{n}}$ superlattices (Fig 1) are constructed by integrating the ferroelectric, antiferromagnetic $\mathrm{LuFeO}_{3}$ and paraelectric, ferrimagnetic $\mathrm{LuFe}_{2} \mathrm{O}_{4}$. The hexagonal $\mathrm{LuFeO}_{3}$ is an improper ferroelectric where the Lu-O buckles into a polar structure [1]. We quantify the polar structure - manifest as a displacement of the lutetium atoms - with atomic precision for different superlattice layerings. Our ferroelectric domain measurements show large polarization and regular domain walls correlate with improved magnetic moment and critical temperature, $\mathrm{T}_{\mathrm{C}}$.

Aberration-corrected scanning transmission electron microscopy (STEM) can provide atomic column positions with sub-picometer precision [2]. Here we apply atomic mapping with picometer precision to find local polarization and domain structure in $\left(\mathrm{LuFeO}_{3}\right)_{\mathrm{m}}\left(\mathrm{LuFe}_{2} \mathrm{O}_{4}\right)_{\mathrm{n}}$ as a function of $m, n$ in a $100 \mathrm{keV}$ NION Ultra-STEM. Each image was formed from many $(>20)$ cross-correlated fast acquisitions. 2D Gaussian fitting located the center of the lutetium columns, from which the displacement, and thereby polarization, was measured (Fig 2ab). After analyzing over 100 images, or $>4000 \mathrm{~nm}^{2}$ of the material, we find that the $\left(\mathrm{LuFeO}_{3}\right)_{\mathrm{m}}\left(\mathrm{LuFe}_{2} \mathrm{O}_{4}\right)_{1}$ superlattices with $m \geq 2$ display a ferroelectric structure. The polarization is damped for lutetium rows adjacent to the $\mathrm{LuFe}_{2} \mathrm{O}_{4}$ layers for $2 \leq m \leq 5$, thus we would expect net polarization to grow with layer width. Indeed, the polarization of the parent-compound $\mathrm{LuFeO}_{3}$ is reached for $m \geq 3$ multilayers and surprisingly is exceeded for $m=7,8$ (Fig $2 \mathrm{c}$ ).

We collected domain sizes and boundary type statistics from the local polarization data. We found that domain size grows with $m$, increasing the width in the growth plane but limited in height by the $\mathrm{LuFe}_{2} \mathrm{O}_{4}$ layers, for up to $m=7$. At $m=7$, the domain height stabilizes at half the height of the $\left(\mathrm{LuFeO}_{3}\right)_{7}$ layer, and forms regular domains with tail-to-tail walls at the $\mathrm{LuFe}_{2} \mathrm{O}_{4}$ layer and head-to-head walls in the middle of the $\left(\mathrm{LuFeO}_{3}\right)_{7}$ layer. First-principle calculations suggest that the doping associated with this particular domain configuration leads to the increase in magnetic moment, which we have observed (Fig 3). $\mathrm{T}_{\mathrm{C}}$ is also seen to increase, approaching room temperature. STEM analysis combined with first-principles results suggests pursuing strongly ferroelectric domains with regular domain boundaries will lead to enhanced magnetization and $\mathrm{T}_{\mathrm{C}}$ in these multiferroics. [3] 
References:

[1] H Das, et al, Nat. Comm. 5 (2014) 2889.

[2] A Yankovich et al, Nat. Comm. 5 (2014) 4155.

[3] Work supported by the U.S. DOE BES, Award \#DE-SC0002334. EM Facility support from the NSF MRSEC program (DMR 1120296).
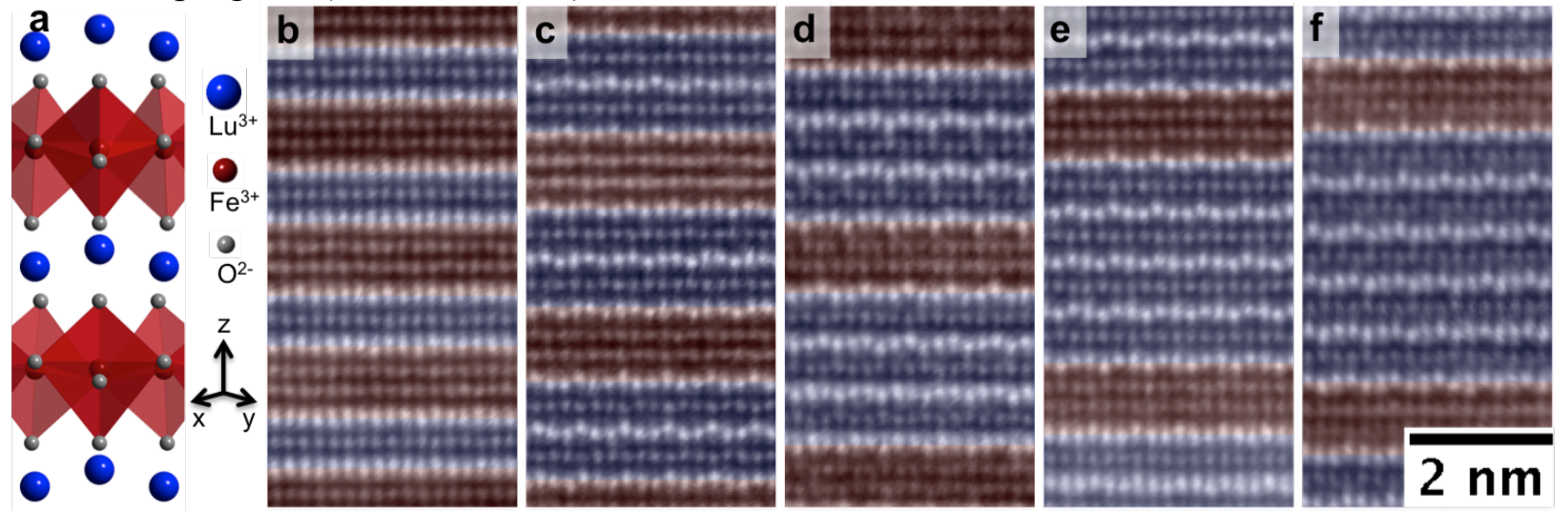

Figure 1. Diagram of the Lu-O buckling in $\mathrm{LuFeO}_{3}(\mathrm{a})$, which leads to the ferroelectric structure [1]. ADF-STEM of the $\left(\mathrm{LuFeO}_{3}\right)_{\mathrm{m}}\left(\mathrm{LuFe}_{2} \mathrm{O}_{4}\right)_{\mathrm{n}}$ viewed along the [110] zone axis, the $\left(\mathrm{LuFeO}_{3}\right)_{n}$ blocks highlighted in blue, for the $m, n=1,1$ structure (b), 2, 1 (c), 3, 1 (d), 4, 1 (e) and 5, 1 (f).
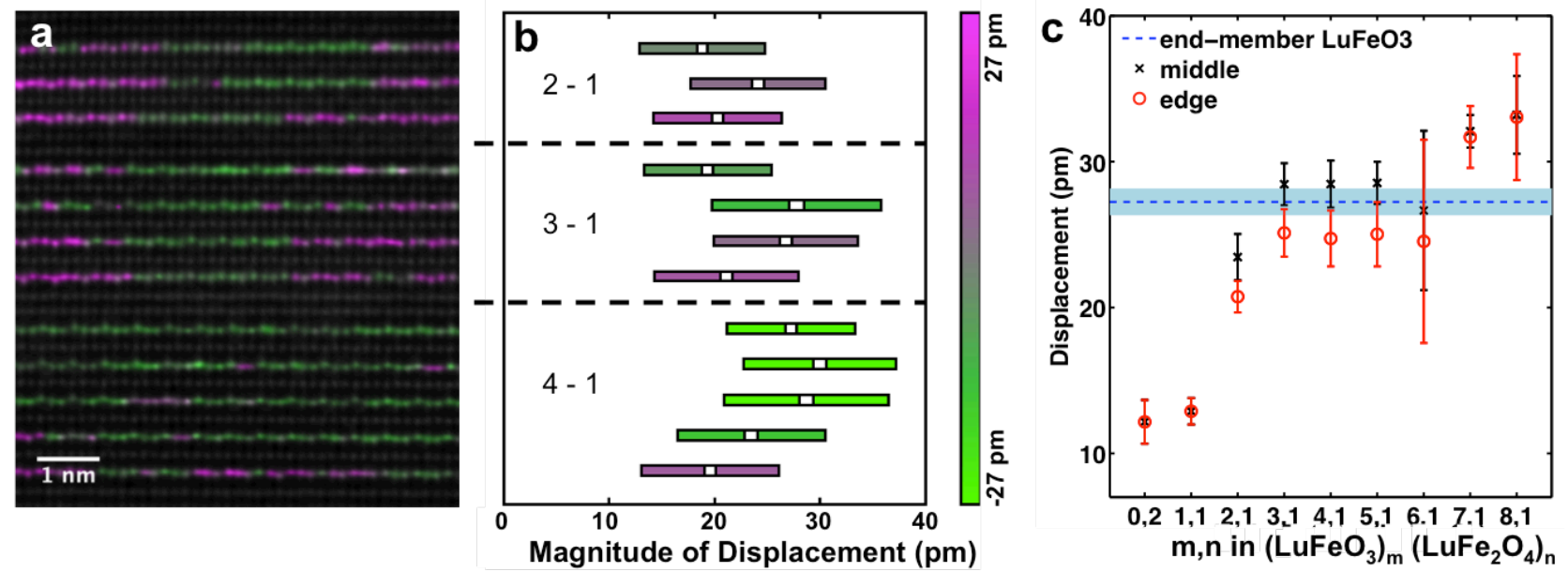

Figure 2. (a) STEM image of $\left(\mathrm{LuFeO}_{3}\right)_{\mathrm{m}}\left(\mathrm{LuFe}_{2} \mathrm{O}_{4}\right)_{\mathrm{n}}$ with color overlays for the magnitude and direction of the local ferroelectric displacements. (b) r.m.s. displacement (square) for each lutetium row. Color indicates mean polarization, with lengths corresponding to $20-80 \%$ of the distribution. (c) r.m.s displacement averaged over many images for varying $(m, n)$, distinguishing between layers on the edge bordering the double iron layer and in the middle. Blue line is for $\mathrm{LuFeO}_{3}$.
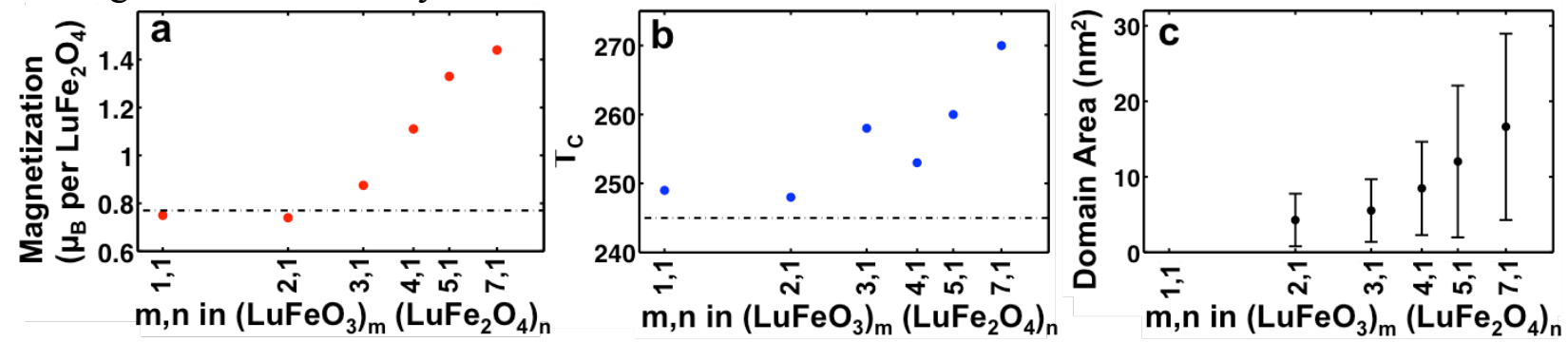

Figure 3. Magnetization and critical temperature for the $\left(\mathrm{LuFeO}_{3}\right)_{\mathrm{m}}\left(\mathrm{LuFe}_{2} \mathrm{O}_{4}\right)_{\mathrm{n}}$, plotted alongside ferroelectric domain area. Larger domain area correlates to higher magnetization. 